\title{
Transposition
}

Musique et Sciences Sociales

Musique : patrimoine immatériel ?

\section{Le fleuve qui fait résonner le son du tambour : musique et patrimonialisation de la folia de reis au Minas Gerais (Brésil)}

The River that Carries the Sound of the Drum: Music and Heritagization of Folia de Reis in Minas Gerais (Brazil)

\section{Lúcia Campos}

\section{OpenEdition}

Journals

Édition électronique

URL : http://journals.openedition.org/transposition/2919

DOI : 10.4000/transposition.2919

ISSN : 2110-6134

Éditeur

CRAL - Centre de recherche sur les arts et le langage

Référence électronique

Lúcia Campos, «Le fleuve qui fait résonner le son du tambour : musique et patrimonialisation de la folia de reis au Minas Gerais (Brésil) », Transposition [En ligne], 8 | 2019, mis en ligne le 15 septembre 2019, consulté le 17 décembre 2020. URL : http://journals.openedition.org/transposition/2919 ; DOI : https://doi.org/10.4000/transposition.2919

Ce document a été généré automatiquement le 17 décembre 2020.

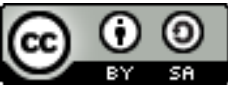

La revue Transposition est mise à disposition selon les termes de la Licence Creative Commons Attribution - Partage dans les Mêmes Conditions 4.0 International. 


\title{
Le fleuve qui fait résonner le son du tambour : musique et patrimonialisation de la folia de reis au Minas Gerais (Brésil)
}

\author{
The River that Carries the Sound of the Drum: Music and Heritagization of Folia \\ de Reis in Minas Gerais (Brazil)
}

Lúcia Campos

1 Le Minas Gerais est un État brésilien reconnu pour son patrimoine historique, à savoir ses églises anciennes, construites au XVIII ${ }^{e}$ siècle, au temps où l'or érigeait des villes et soutenait la circulation des biens, des personnes et des imaginaires. La région est également réputée pour son patrimoine naturel, ses paysages et formations géologiques, témoins de peuples considérés comme les premiers habitants des Amériques. C'est aussi un territoire marqué par l'exploitation minière, et qui a récemment été victime de crimes environnementaux aux proportions alarmantes. Entre églises, grottes, villes, patrimoine architectural et patrimoine naturel, entre les menaces et le pouvoir économique de l'exploitation minière, existent et résistent dans tout l'État des groupes dits de " culture populaire », terme qui comprend les savoirs et les actions de ce que nous appelons aujourd'hui le patrimoine culturel immatériel.

2 Cet article s'intéressera à l'approche du patrimoine culturel dans cet État si intensément marqué par une conception historique, architecturale, et donc éminemment matérielle, du patrimoine. Il s'agira de comprendre comment la catégorie de "patrimoine culturel immatériel " s'insère dans ce contexte, surtout à travers les politiques publiques locales issues de l'IEPHA ${ }^{1}$, qui est l'organisme responsable de la protection du patrimoine historique et artistique du Minas Gerais. À cette fin, nous présenterons les récentes interventions de l'État dans le domaine du patrimoine culturel immatériel, et mettrons l'accent sur la patrimonialisation d'une pratique religieuse appelée folia de reis, à la présentation de laquelle est dédiée la première partie de cet article. Les pratiques musicales sont fondamentales dans les groupes de folia de 
reis, ce qui confère à la transmission musicale un rôle décisif dans la préservation de cette pratique. Nous traiterons ensuite du décalage entre les manières qu'ont respectivement les groupes de folia de reis et les pouvoirs publics d'envisager le rôle de la «musique ».

Notre enquête, fondée sur une ethnographie d'un groupe de folia de reis de Lagoa Santa ${ }^{2}$, la Folia de Reis de São Sebastião do Campo Belo, étudie la manière dont les formes de transmission musicale entre différentes générations assurent la persistance de cette pratique depuis plus d'un siècle ${ }^{3}$. Du fait de la récente déclaration des folias comme patrimoine culturel immatériel par le Conselho Estadual do Patrimônio Cultural, cette enquête vise aussi à évaluer les rapports que les folias entretiennent avec les pouvoirs publics $^{4}$ : comment les maîtres et les participants des folias, c'est à dire, les foliões, entendent-ils la catégorie "patrimoine immatériel»? Quel est l'impact de cette nouvelle catégorie normative sur les pratiques et comment peut-elle intervenir dans la préservation de sa transmission?

\section{Folia de reis : " une joie dans la maison de chacun »}

Il y a le drapeau des trois rois. Notre-Dame, Saint Joseph et l'enfant Jésus, et Saint Sébastien. Sans le drapeau on ne marche pas, on ne sort même pas de chez soi. À la fin de l'année, on laisse le drapeau dans une maison, l'année suivante on va le chercher dans la maison où on l'a laissé. (Pedro Henriques Rodrigues, Folia de Reis de São Sebastião do Campo Belo, Lagoa Santa, $\mathrm{MG}^{5}$ ).

4 En décembre et janvier, dans plusieurs régions du Brésil, des groupes de folia de reis circulent et maintiennent vivante une pratique religieuse qui dure depuis des générations. Ils sont particulièrement nombreux au sud-est du pays, tant dans les zones rurales qu'urbaines ${ }^{6}$. Le pèlerinage des folias, généralement appelé giro (tour), se déroule de maison en maison, où les participants saluent les habitants et révèrent les crèches de Noël, dans une célébration chantée, jouée et dansée. Selon Pedro Henriques Rodrigues, coordinateur et trésorier de la Folia de Reis de São Sebastião de Campo Belo, " c'est une joie dans la maison de chacun ${ }^{7}$ ".

5 Les folias de reis sont des associations communautaires à caractère religieux, participant du catholicisme populaire, qui mettent en scène (avec musique, danse et poésie) le parcours mythique des trois rois mages entre Noël et l'Épiphanie. L'ethnomusicologue Suzel Ana Reily, qui a beaucoup travaillé sur les folias du sud du Minas Gerais, affirme que « la tradition se fonde sur une conception populaire selon laquelle les rois mages seraient devenus des musiciens ${ }^{8} »$. La musique constitue dès lors le socle d'organisation des groupes, dont la formation vocale varie selon la région. Elle est jouée généralement par des instruments à percussion tels que les caixas (caisses) et pandeiros (tambours sur cadre), et sur des instruments harmoniques tels que les violas (guitares à dix ou douze cordes), guitares à six cordes, cavaquinhos (petites guitares à quatre cordes) et accordéons. D’après Reily, le style musical le plus répandu dans les folias du sud-est est le style "mineiro" (du Minas). Selon l'auteur, "ce style emploie de cinq à huit voix distinctes qui intègrent l'ensemble de manière cumulative ${ }^{9} »$. Bien que les folias du Minas Gerais représentent un héritage partagé dans l'espace et le temps, il est important de souligner l'autonomie de chaque groupe, qui finit par développer ses propres caractéristiques musicales. 
6 En racontant l'histoire de la folia de reis do Campo Belo que son père dirigeait, Pedro Henriques Rodrigues décrit la folia qu'il a connue à la campagne, qui va de maison en maison. Il nous dit notamment qu'il est difficile de maintenir la folia en ville, où tout le monde est affairé avec son travail et ses obligations, et manque de temps et d'intérêt. Geraldo, le cousin de Pedro, est aussi l'un des coordinateurs de la folia do Campo Belo ; tous deux sont maçons dans la région de Lagoa Santa. Geraldo dit qu'il a appris la folia avec son père, qui lui a enseigné plus de vingt-cinq vers ${ }^{10}$ transcrits par ses soins, auxquels se greffent ses improvisations.

7 Geraldo et Pedro se souviennent avec plaisir des folias à la campagne, qu'ils préfèrent en raison de l'accueil qui leur est réservé par les hôtes de chaque maison. En ville, la mairie ne les aide pas et ils aiment autant se tenir à distance des pouvoirs publics : "C'est comme ça depuis cinquante ans, pourquoi faire autrement ${ }^{11}$ ? " Ils vont là où on les invite, "même chez des croyants (d'autres religions) ${ }^{12}$ ». Ils se plaignent parce que les églises évangéliques interdisent à d'anciens habitués de participer à la folia. Certains ont dû la quitter. Malgré leurs difficultés (qu'ils sont unanimes à souligner), ils sont fiers de garder en vie la folia héritée de leurs parents.

Si tu n'es pas prêt à pardonner, tu ne restes pas à la folia. Si ton cœur n'est pas préparé, il ne faut pas y aller. Avec tout le malheur qui arrive au monde aujourd'hui, la folia en marche porte bonheur. Elle est déjà entrée dans une maison où il y avait un chien enragé qui pourtant n'a attaqué personne. Bœuf enragé qui traverse les broussailles, le bœuf ne sauta jamais sur la fête, ne blessa jamais personne. Personne ne s'est jamais cassé une jambe ou un bras en marchant derrière la folia. Et le bœuf l'accompagne. Tu joues d'un instrument et le bétail se rassemble. D'abord ils courent, puis ils écoutent le son de la viola et se rassemblent. Ou alors le son d'un tambour, et tout le bétail se rassemble. Quand est frappé un tambour au bord du fleuve des Velhas ${ }^{13}$, on entend le bruit du tambour cinq kilomètres en aval. Les eaux transportent le son. La folia a quelque chose... Parfois je m'en souviens et je pleure, c'est si bon ${ }^{14}$.

Ces personnes maintiennent une cohésion en vivant une religion dansée, jouée et chantée. L'architecture de sept voix superposées crée un temple au moment du partage. Un temple mobile, itinérant, puissant, sonore. Un temple immatériel.

\section{Enjeux de la patrimonialisation de l'immatériel au Minas Gerais}

Depuis 2016, l'Instituto Estadual do Patrimônio Histórico e Artístico de Minas Gerais IEPHA - procède au recensement des folias de reis de la région, afin d'établir une cartographie des groupes et de promouvoir des projets de sauvegarde des pratiques. L'initiative relève des dispositions mises en place en 2002, deux ans après la déclaration fédérale en faveur du patrimoine culturel immatériel. Après la première déclaration, qui concernait les procédés de fabrication du fromage du Minas Gerais, l'IEPHA s'attache à reconnaître des fêtes et des célébrations comme relevant du patrimoine culturel immatériel de la région. La direction actuelle de cet organisme a vu dans la patrimonialisation de la folia de reis, pratique largement présente sur le territoire du Minas Gerais, la possibilité de développer et de promouvoir l'unité de la région.

10 L'inventaire des folias de reis a été réalisé via une plateforme collaborative par les groupes eux-mêmes ou par les mairies de chaque municipalité. Ce travail de l'IEPHA, commencé en 2016, a abouti au recensement de plus de 1500 groupes de différentes 
régions du Minas Gerais. Le processus est toujours en cours : les inscriptions dans la base se poursuivent, en flux constant, pour inclure tous les groupes concernés. Il est important de souligner que la folia de reis n'était pas en péril de disparition. Ce n'est pas un « patrimoine en danger ", mais un patrimoine qui devient représentatif de la région.

11 S'agissant des propositions de sauvegarde, la gestion du patrimoine du Minas Gerais jouit d'un instrument important, l'ICMS ${ }^{15}$, l'impôt du patrimoine, qui permet un financement local des actions. La gestion de l'IEPHA vise la collaboration entre les municipalités pour donner de la visibilité aux biens culturels. D'après les recherches réalisées par l'institution, les principales demandes des groupes de folias de reis concernent des instruments, des vêtements et des transports. La solution proposée en réponse à ces demandes passe par des appels d'offre spécifiques dans le domaine du patrimoine, calqués sur d'autres appels déjà existants pour les productions musicales. Mais l'un des principaux buts que se donnent les politiques est de constituer des réseaux et d'en mobiliser les participants à travers l'organisation de rencontres entre les groupes des différentes villes; nous nous demanderons plus loin dans quelle mesure ces groupes eux-mêmes sont intéressés par ce type de mobilisation.

La reconnaissance des folias de reis comme « patrimoine culturel immatériel » du Minas Gerais, opérée à partir d'un inventaire des groupes de la région, s'insère dans un mouvement international de valorisation de cette nouvelle catégorie patrimoniale qui cherche à intégrer la dimension immatérielle et prendre en compte les valeurs sociales du patrimoine ${ }^{16}$. Si cette catégorie vise la préservation de manifestations culturelles de tradition orale dans le monde, plusieurs anthropologues en critiquent les dérives, dès lors que cette notion peut conduire à la sauvegarde non de la culture vivante, mais de ses représentations ${ }^{17}$. Il convient dès lors de mettre en relief les différences entre les groupes de folias de reis et les politiques du patrimoine culturel immatériel quant à leurs conceptions et usages respectifs de la musique. Au-delà de l'importance déjà soulignée de la musique - chanter, jouer d'un instrument, danser -, qui doit se traduire dans les politiques patrimoniales, l'idée de "musique" elle-même pose question dans ce contexte: quels formats de rencontre et de représentation suppose-t-on? Quelles logiques d'organisation, quels modes d'écoute? S'agirait-il de transformer les folias de reis en groupes de musique comme n'importe quels autres?

\section{« La folia n'est pas une bringue »}

13 Avant le giro, qui se passe du jour de Noël à la mi-janvier, la folia de reis de Campo Belo fait quelques répétitions au mois de novembre, afin de rassembler le groupe et d'organiser la partie musicale : on vérifie l'état de chaque instrument, on décide qui va jouer de quel instrument, on se répartit les voix. L'importance attribuée à l'organisation des voix par Pedro, par Geraldo et par les autres membres de la folia do Campo Belo suggère que la fonction musicale est structurelle pour ce groupe.

Pendant les répétitions alternent des rythmes d'origine afro-brésilienne: pagodes, lundus, batuques, maxixes. Les participants chantent en chœur ou sous forme responsoriale (un personnage chante, et le chœur lui répond). Les sept voix sont là, chacune prise en charge par un chanteur. Pedro Henriques m'explique que, dans la folia, la viola est l'instrument le plus important - les instruments à cordes, en général, sont très importants. La Folia de Reis do Campo Belo est composée de cordes - viola et cavaquinhos - et d'une caixa (caisse). Il montre la caisse "sourde », une caisse ancienne, 
et dit du son qu'il doit être "sourd", c'est à dire étouffé. Il montre à l'arrière les hochets de serpent attachés à une corde, qui produisent une sonorité caractéristique. Le joueur de cette caisse est l'un des piliers de cette folia. Le son est ferme, présent, fort. Il donne la cadence nécessaire pour les autres instruments, pour les voix et pour la danse. C'est lui que les personnages dansants regardent. Ils chantent et interagissent avec lui, les yeux dans les yeux.

Les répétitions sont un lieu d'activation de la mémoire et surtout de transmission. Les plus jeunes imitent les plus âgés. Celui qui joue apprend aussi des plus expérimentés. Les adultes, les jeunes et les enfants dansent. La transmission devient plus évidente dans la danse. Les enfants incorporent les personnages, ils dansent et s'arrêtent pour chanter. Ils ne reproduisent pas toujours bien les marches, ils se trompent, mais ils sont là et leurs efforts pour apprendre sont valorisés par le groupe.

La mémoire de Pedro semble être la force qui anime le groupe. Il cherche les sons, les voix, les gestes, les gens, pour recréer la performance qu'il a en mémoire, ce qui, à cinquante-cinq ans, est loin d'être évident - il se dit fatigué. Mais c'est ce fil de mémoire qui soutient la rencontre et la religiosité présente. "La folia n'est pas une bringue ${ }^{18}$ ", me dit-il. On boit, on danse, il y a de la musique, de la célébration, mais « ce n'est pas une bringue ».

En décrivant le rituel du règne de Notre-Dame du Rosaire au Minas Gerais, l'ethnomusicologue Glaura Lucas souligne l'importance de la musique pour la cohésion religieuse des groupes et explique en quoi la foi fonde l'action :

Prendre part à la musique, ainsi qu'au rituel dans son ensemble, c'est d'abord un acte de foi et donc une source de plaisir, de satisfaction et de joie, et qui ressemble davantage à la réalisation d'un travail ou à l'accomplissement d'une mission pouvant impliquer également des sacrifices sous la forme de souffrances personnelles - qu'à un divertissement ${ }^{19}$.

Elle affirme encore que «la musique, organisant l'interaction coordonnée des gestes collectifs dans le temps, joue un rôle important pour former l'union du groupe, renforce les liens d'appartenance à la tradition et la réaffirmation et négociation des valeurs spirituelles, culturelles et communautaires ${ }^{20}$ ». Concernant les folias de reis, l'ethnomusicologue Suzel Reily souligne également le rôle de la musique pour organiser les actions et les interactions des participants, dans le but de «forger un sens de l'expérience partagée ${ }^{21} »$.

19 Pedro cherche à faire comprendre que ce qui anime la folia n'est pas la fête, mais la foi, la religion. Il dit que le goût et la joie soutiennent la folia, mais aussi que cette dernière " exige un très grand effort physique, la voix doit être très forte ${ }^{22}$ ». Il insiste encore pour dire que « la folia n'est pas une bringue ». Le goût, le plaisir et la joie proviennent de la relation entre les gens, relation structurée par le chant, par la danse, par la prière, par l'organisation particulière autour des personnages et du giro de maison en maison. La religion est ici organisée de manière itinérante à travers la musique, c'est-à-dire que musique et religion se structurent mutuellement - d'où notre idée d'un "temple immatériel » dont les fondements reposent sur les relations à la fois musicales et religieuses qui s'y sont construites. 


\section{Quelles médiations possibles ? - ou le risque de musicaliser le patrimoine culturel immatériel}

La pratique religieuse de la folia de reis présente une organisation interne, avec pour base le récit des trois rois mages, ses personnages, ses chants et danses respectifs. Elle arbore aussi une structure temporelle et spatiale autour du giro entre les maisons de la communauté concernée par la célébration, alternant des moments dans l'espace public des rues et d'autres dans l'espace privé des maisons. Pedro Henriques est assez réticent devant les initiatives des pouvoirs publics concernant les folias. Il nous fait part d'invitations qu'il a reçues de la mairie de Lagoa Santa à des «rencontres de folias» («encontros de folias») et d'autres présentations sur scène, en dehors de l'époque du giro. D'après lui, ces rencontres n'ont pas été bien reçues par le groupe et ont donné lieu à des malentendus concernant les objectifs d'une folia de reis :

Il n'y a pas d'enthousiasme, c'est hors de propos. On ne reçoit rien, on ne demande rien, parfois seul le transport est pris en charge. Il n'y a pas d'enthousiasme car il n'y a personne sur place pour recevoir la folia. On nous y a fait venir pour faire la fête, mais ce n'est pas le but, ce n'est pas exactement une fête, notre but c'est d'apporter une joie dans la maison de chacun. Dans son périple par le monde, Jésus ne demandait pas de fêtes, il allait de maison en maison. Je n'aime pas m'attarder dans une maison avec la folia, ce n'est pas correct. Dans notre conception ce n'est pas correct ${ }^{23}$.

Attaché à la fonction religieuse et musicale du giro, Pedro Henriques ne voit pas d'un bon œil les présentations de la folia de reis en dehors du calendrier religieux qui lui est propre, en dehors de l'ordre rituel qui en régit l'organisation. Les prestations animées d'intentions autres que la célébration de la Nativité ne sont pas de son goût. Il existe en effet une grande différence entre ce que l'on peut entendre par « fête » et ce que Pedro Henriques comprend comme "une joie dans la maison de chacun». Il estime que les présentations aléatoires en public n'ont pas de sens, et refuse les éventuels financements offerts par la mairie, dont l'obtention est selon lui entravée par la complexité des démarches administratives à entreprendre. Il préfère ne pas compter sur ces subventions censées soutenir le processus de patrimonialisation et se désintéresse de l'inscription des folias de reis comme patrimoine, promue par l'IEPHA. Et d'affirmer: "notre subvention à nous, c'est notre cœur, notre volonté, notre courage ${ }^{24} »$.

L'anthropologue José Reginaldo Gonçalves affirme la nécessité de qualifier la catégorie de « patrimoine » en " explorant ses conceptions natives et donc en nous éloignant de ses usages idéologiques et légaux ». Nous considérons avec lui que, d'un point de vue natif,

[...] les patrimoines existent toujours dans des formes matérielles spécifiques, et sont construits et perçus à travers des codes sensitifs - vision, toucher, odorat, audition, goût - et non comme des formulations purement abstraites appartenant à des discours programmatiques. [...] Ils ont l'existence d'objets matériels situés dans des espaces aux modalités spécifiques, inséparables de l'expérience humaine et donc des "techniques du corps». En ce sens, il est impossible d'étudier le «patrimoine immatériel » sans s'intéresser de près à ses dimensions éminemment matérielles, ses usages sociaux et individuels, ainsi qu'à ses productions et à ses effets $^{25}$.

L'étude de la patrimonialisation de la folia de reis invite donc à s'intéresser de près à ce que les foliões comprennent comme leur «patrimoine ». L'ethnographie montre qu'ils 
défendent une notion de patrimoine liée à la mémoire et aux liens communautaires établis entre musique et religion, dimensions qui s'articulent entre elles, qui entourent des personnes, des pratiques, des gestes, des voix, des vers, des instruments, des vêtements, des lieux spécifiques et qui, dans leur conception, ne peuvent être séparés. Mais la catégorie "patrimoine " n'apparait, de façon plus explicite, que quand ils l'utilisent comme une "catégorie d'aller-retour ${ }^{26}$ ", c'est à dire, quand ils s'approprient cette catégorie pour faire la médiation entre différentes instances et pour défendre les spécificités de leur pratique religieuse et communautaire auprès des pouvoirs publics.

Comme nous l'avons vu, les propositions de sauvegarde du patrimoine du Minas Gerais s'appuient sur des impôts qui subventionnent localement des politiques publiques pour ce domaine. Dans ce cadre, l'articulation entre l'implication des municipalités et la création de « réseaux » est l'un des points forts, qui entend donner une "visibilité au bien culturel »; c'est cette recherche de "visibilité » qui régit l'organisation des "rencontres de folias». Cependant, les groupes ne sont pas toujours intéressés par de tels événements. Malgré son importance, la "musique», dans sa conception qui est mobilisée dans les politiques de sauvegarde, se limite parfois à des formats de rencontre et de présentation qui renvoient à des paradigmes d'organisation et des modes d'écoute issus de "mondes de l'art ${ }^{27}$ ». Or ces derniers sont externes, voire étrangers à une grande partie des groupes: on assiste au passage d'une "musiquerelation" à une "musique-objet ${ }^{28}$ ». Autrement dit, le processus tend bien à transformer les folias de reis en des groupes musicaux comme les autres.

Dans d'autres études menées au Brésil sur la circulation internationale d'un groupe de samba de roda de Bahia ${ }^{29}$, pratique musicale brésilienne déclarée patrimoine culturel immatériel par l'UNESCO, et sur les maîtres du maracatu de baque solto du Pernambuco, depuis le carnaval jusqu'au festivals de musiques du monde ${ }^{30}$, nous avons pu aborder la relation entre des groupes dits de cultura popular et les scènes musicales. Dans ces deux cas, le passage à la scène et l'enregistrement de disques, parmi d'autres procédures, impliquaient des transformations des dispositifs, des formats et des modes d'écoute. Néanmoins, ces processus, ayant comme protagonistes les acteurs eux-mêmes ou des médiateurs, étaient enclenchés avant la patrimonialisation: les pratiques sonores et musicales étaient déjà tenues pour des " objets ", présentées dans des concerts avec la médiation de producteurs. Dans le cas du samba de roda, la production musicale fut d'ailleurs l'un des axes du plan de sauvegarde, élaboré par les groupes avec la médiation de chercheurs ${ }^{31}$.

Les folias de reis du Minas Gerais, en revanche, deviennent des objets de négociation à partir des formats de production culturelle, sans que les acteurs soient tenus pour protagonistes de la reconnaissance de ce qu'ils entendent localement comme leur " patrimoine ». Dans ce contexte, l'évènement et la production culturelle deviennent des mesures superficielles de sauvegarde. On peut alors se demander ce que l'on cherche ici à préserver entre les pratiques et leurs représentations médiatiques.

\section{Limites et intersections entre le matériel et l'immatériel}

À travers l'analyse de la patrimonialisation et des mesures de sauvegarde de la folia de reis, on perçoit un processus qui tend au formatage de certaines pratiques musicales, 
que l'on peut critiquer en tant qu'il impose les critères de la «musique-objet». Ce processus réifie l'immatériel, allant de l'inventaire à la médiatisation sans passer par une recherche-action capable de prendre en compte les conceptions quotidiennes des folióes et de partager effectivement avec ces derniers une gestion collaborative des mesures de sauvegarde.

Dans le cas de la folia de reis, ce processus de réification a pris une tournure encore plus radicale. Après l'inventaire et l'inscription des folias comme patrimoine immatériel du Minas Gerais, les désirs patrimoniaux de l'IEPHA se sont tournés vers un instrument musical qui présente un rôle central dans les folias de reis, la viola, à la reconnaissance de laquelle ils ont contribué. Comment comprendre ce chemin pris par les politiques patrimoniales, de la fête à un élément matériel, un instrument musical, qui est partie intégrante et structurante de cette fête ? Le passage d'un processus à l'autre, de la folia à la viola comme "patrimoines ", montre là encore une tendance à la réification de la pratique, selon les critères de la «musique-objet ».

Au gré d'un entretien, Michele Arroyo, présidente de l'IEPHA, nous fournit quelques clés au sujet de la relation entre le matériel et l'immatériel dans le processus de patrimonialisation en cours. Elle défend l'idée que «la séparation fut nécessaire pour prendre position, pour porter un regard sur le patrimoine comme processus et non comme quelque chose de statique », et considère que «la jonction de la matérialité et de l'immatérialité va commencer à se faire à partir des actions de sauvegarde et non nécessairement des actions de reconnaissance, car il n'est pas possible de préserver si l'on ne travaille pas la matérialité et l'immatérialité de ce fait culturel $\$^{32}$. La situation est paradoxale : d'un côté, la patrimonialisation de la viola peut en effet conduire à une meilleure reconnaissance des instruments dits «populaires » dans les médias et dans l'enseignement officiel; néanmoins, d'un autre côté, elle peut tendre à réduire la folia de reis à l'un de ses instruments, donc à un seul de ses éléments musicaux, laissant de côté les enjeux sociaux et religieux qui la structurent.

30 L'institution de la viola comme patrimoine souligne le besoin de penser selon la perception native du patrimoine défendue par Gonçalves, en considérant non seulement les éléments matériels, mais aussi les gestes, les sons, les écoutes qui entourent la construction et l'exécution des instruments musicaux. Or, le passage de la reconnaissance d'une « fête » à l'un de ses instruments de musique montre encore une fois le rôle central des savoir-faire musicaux dans la structuration de la célébration. Un tel passage nous amène à réfléchir à d'autres chemins possibles pour penser la sauvegarde des savoir-faire musicaux dans le quotidien des communautés concernées.

\section{L'immatériel comme processus : comment penser le patrimoine culturel au quotidien?}

31 En se référant à « des processus [qui] ont cessé de contempler presque exclusivement des reconstructions du passé pour s'intéresser à des manifestations culturelles vivantes et vibrantes comme les fêtes, rituels, savoirs, connaissances traditionnelles", l'anthropologue Regina Abreu affirme que

ce changement de statut des biens culturels, quand ils passent par le processus de patrimonialisation, implique aussi des changements de perception, de nouveaux transits et la transformation des visions du monde, dans une perspective dialogique entre différents agents ${ }^{33}$. 
32

donne l'exemple de la patrimonialisation par l'UNESCO des peintures Kusiwa des Amérindiens de l'ethnie Wajãpi, au Brésil, qui en viennent à entretenir deux modes de relation avec les « biens culturels » :

D'un côté, dans le vécu, en maintenant la tradition en circulation dans le groupe et dans la mémoire sociale et, d'un autre côté, en la systématisant dans les discours, c'est-à-dire en représentant, par le moyen des langages audiovisuels, photographiques, numériques, des aspects de cette tradition qui doivent être permanents ${ }^{34}$.

Comme l'affirme l'anthropologue, "dans ce cas, le "bien culturel" ne sort pas de la circulation, c'est-à-dire qu'il ne perd pas sa "valeur d'usage" [...]. Mais, effectivement, il acquiert une valeur symbolique qu'il n'avait pas auparavant, même s'il continue à être amplement utilisé au quotidien ${ }^{35}$ ». Ainsi, la patrimonialisation a trait à une dimension valorisante même si l'activité consacrée appartient à la vie quotidienne des communautés, et que l'on ne veut pas - en principe - changer cette activité quotidienne. Abreu confère alors à l'espace scolaire un rôle fondamental pour saisir les défis que la systématisation discursive d'un tel bien impliquerait. médiatiques veut montrer également la nécessité de penser à des articulations plus consistantes et plus durables dans le quotidien des communautés concernées. L'articulation dialogique entre des études, des recherches et la pratique de l'éducation patrimoniale pourrait-elle en être une voie? Les défis de la patrimonialisation de l'immatériel montrent que l'école peut être un espace crucial pour donner consistance aux plans de sauvegarde, afin de comprendre les savoir-faire en question dans le quotidien communautaire et au-delà, comme représentatifs de telle communauté en contact avec la sphère publique nationale. Certes, pour que l'école soit le lieu où ces différents mondes entreraient en relation dialogique, l'éducation patrimoniale doit être pensée à partir des savoirs partagés par la communauté, à partir des inventaires participatifs, les maîtres étant médiateurs de leur propre savoir.

Il devient également nécessaire de produire une connaissance plus approfondie des pratiques patrimonialisées, fondée sur des recherches ethnographiques des folias de reis et aussi des recherches-participatives avec les foliões, qui puissent situer et problématiser l'impact de la patrimonialisation. Particulièrement dans le cas des folias de reis, il n'existe pas d'articulation claire et continue entre le cadre universitaire, dans lequel la plupart des recherches sont réalisées, et les politiques publiques de l'IEPHA ${ }^{36}$.

Comme nous le suggère le propos du folião Pedro Henriques, la patrimonialisation pourrait finalement être considérée comme le fleuve qui fait résonner le son du tambour: "Quand est frappé un tambour au bord du fleuve des Velhas, on entend le bruit du tambour cinq kilomètres en aval. Les eaux transportent le son... » Reconnaître la folia de reis comme patrimoine culturel, c'est écouter les personnes qui pratiquent la folia, les chercheurs, les institutions de sauvegarde, les écoles; c'est prêter l'oreille à tout ce qui contribue à faire la folia et à la constituer en patrimoine. C'est faire l'expérience de cette écoute qui est celle du tambour, mais aussi celle du fleuve. 


\section{BIBLIOGRAPHIE}

ABREU Regina, « Patrimonialização das diferenças e os novos sujeitos de direito coletivo no Brasil », TARDY Cécile, DODEBEL Vera (dir.), Memória e Novos Patrimônios, Marseille, OpenEdition Press, 2015.

BECKER Howard, Les mondes de l'art, Paris, Flammarion, 1998 [1982].

BITTER Daniel, « Versos de improvisos nas chulas de palhaços de folias de reis », PIMENTEL Alexandre, CORREA Joana (dir.). Na ponta do verso : poesia de improviso no Brasil, Rio de Janeiro, Associação Cultural Caburé, 2008, p. 102-117.

BORTOLOTTO Chiara, Le patrimoine culturel immatériel : enjeux d'une nouvelle catégorie, Paris, Éditions de la Maison des sciences de l'homme, 2011.

CAMPOS Lúcia, « Sauvegarder une pratique musicale ? Une ethnographie du samba de roda à la World Music Expo », Cahiers d'Ethnomusicologie, vol. 24, 2010, p. 141-153.

CAMPOS Lúcia, Les modes d'écoute d'une poésie chantée : le maracatu de baque solto de la cultura popular à la scène musicale globalisée, Paris, École des Hautes Études en Sciences Sociales, Thèse de Doctorat, 2016.

CARNeIRo DA CUNHA Manuela, Cultura com aspas e outros ensaios, São Paulo, Cosac Naify, 2009. GONÇALVES José Reginaldo, «The Dark Side of the Moon: Heritage, Memory and Place in Rio de Janeiro, Brazil », Vibrant, vol. 10, nº 1, 2013, p. 190-207.

HENNION Antoine, La Passion musicale : Une sociologie de la médiation, Paris, Edition Métailié, 2007 [1993].

KIRSHEMBLATT-GUIMBLETT Barbara, «Intangible cultural heritage as a meta-cultural production », Museum International, vol. 56, $\mathrm{n}^{\circ}$ 1-2, 2004, p. 52-65.

LUCAS Glaura, Música e tempo nos rituais do congado mineiro dos Arturos e do Jatobá, Rio de Janeiro, Universidade Federal do Estado do Rio de Janeiro, Thèse de Doctorat, 2005, p. 105.

LÜHNING Angela, TUGNY Rosângela (dir.), Etnomusicologia no Brasil, Salvador, EDUFBA, 2016.

REILY Suzel Ana, « As vozes das Folias: um tributo a Elizabeth Travassos Lins », Debates (Unirio), vol. 12, 2014, p. 35-53.

REILY Suzel Ana, Voices of the Magi: Enchanted Journeys in Southeast Brazil, Chicago, Chicago University Press, 2002, p. 14.

SANDRONI Carlos, «L'ethnomusicologue en médiateur du processus patrimonial : le cas du samba de roda ", BORTOLOTTO Chiara, Le patrimoine culturel immatériel : enjeux d'une nouvelle catégorie, Paris, Éditions de la Maison des sciences de l'homme, 2011.

\section{NOTES}

1. Institut du Minas Gerais pour le Patrimoine Historique et Artistique, fondation liée au Secrétariat de la Culture, Minas Gerais, Brésil, chargée de la protection, de la conservation et de la promotion du patrimoine matériel et immatériel du Minas Gerais, suivant les politiques publiques du patrimoine des gouvernements local et national. 
2. Lagoa Santa est un village situé dans la métropole de Belo Horizonte, capitale du Minas Gerais. Le village se situe à 800 mètres d'altitude, avec $231,9 \mathrm{~km}^{2}$ de surface et une population d'environ 62000 habitants en juillet 2017.

3. Nous avons suivi la Folia de Reis de São Sebastião do Campo Belo de novembre 2017 à janvier 2018, et à nouveau de décembre 2018 à janvier 2019. Entre-temps, nous avons réalisé des entretiens avec les participants de ce groupe, notamment avec le coordinateur Pedro Henriques et avec son cousin Geraldo, en charge de la partie musicale. Pour cette recherche, nous avons également suivi d'autres groupes de la région, qui ne sont pas directement abordés dans ce texte. 4. Notre enquête a également porté sur l'IEPHA, à la faveur d'entretiens réalisés avec la présidente de l'institution, Michelle Arroyo, et la directrice de protection et mémoire Françoise Souza, et de l'étude des dossiers des processus de patrimonialisation.

5. «Tem a bandeira dos três reis. Nossa Senhora, São José e o menino jesus, e São Sebastião. Sem a bandeira nos não anda não, nem sai de casa! Chega fim do ano entrega a bandeira numa casa, chega no outro ano, vai buscar, sai daquela casa em que deixou a bandeira. » Entretien du 2 juin 2018 (nous traduisons). Les extraits suivants proviennent du même entretien.

6. BITTER Daniel, "Versos de improvisos nas chulas de palhaços de folias de reis ", PIMENTEL Alexandre, CORREA Joana (dir.), Na ponta do verso: poesia de improviso no Brasil, Rio de Janeiro, Associação Cultural Caburé, 2008, p. 102-117.

7. «É uma alegria na casa de cada um. "

8. «A tradição se fundamenta numa concepção popular que sustenta que os Três Reis Magos teriam se tornado músicos. » REILY Suzel Ana, « As vozes das Folias: um tributo a Elizabeth Travassos Lins », Debates [Unirio], vol. 12, 2014, p. 35-53, ici p. 39.

9. « este estilo emprega de cinco a oito vozes distintas que integram o conjunto de forma acumulativa» (Ibid., p. 39).

10. Les vers des folias de reis portent principalement sur l'adoration de la crèche de Noël et sur le récit des trois rois, mais il existe également des vers d'arrivée et de départ des maisons ainsi que de salutation - des propriétaires des maisons visitées, entre autres.

11. "É assim há cinquenta anos, por que haveria de ser diferente? »

12. «Até em casa de crente ».

13. Das Velhas, fleuve de $801 \mathrm{~km}$ qui naît dans la région proche de la ville d'Ouro Preto, au Minas Gerais, et finit dans le fleuve São Francisco, l'un des plus longs fleuves du Brésil.

14. « Folia, se você não estiver preparado para perdoar você não prende na folia não. Se não tiver o coração preparado, não precisa ir não. Tanto azar que acontece no mundo agora, a folia andando tem uma coisa que abençoa. Já andou em casa com cachorro brabo, entra nas casas, cachorro nenhum ataca. Boi brabo pelo mato afora, boi nunca pulou na folia, nunca machucou ninguém. Ninguém nunca quebrou uma perna ou um braço atrás da folia. E o boi acompanha folia. Você toca assim no interior, o gado junta todo. Primeiro começa a correr, depois escuta o som da viola, essa caixa bater, junta tudo. Quando você bate uma caixa surda na beira do rio das Velhas, cinco quilômetros rio abaixo, você escuta o barulho da caixa, a água leva o som. A folia tem uma coisa. Às vezes eu lembro ainda choro, é gostoso ".

15. Impôt sur la circulation de marchandises et de services. Dans le Minas Gerais, une partie de l'ICMS est attribuée chaque année à la préservation du patrimoine culturel de l'État, par le transfert de ressources aux municipalités qui préservent leur patrimoine et leurs références culturelles par le biais de politiques publiques pertinentes. L'ICMS culturel encourage les actions visant à protéger les actifs des municipalités en renforçant les secteurs responsables du patrimoine des villes et leurs conseils respectifs dans le cadre d'une action conjointe avec les communautés locales.

16. воRтоцотто Chiara, Le patrimoine culturel immatériel : enjeux d'une nouvelle catégorie, Paris, Éditions de la Maison des sciences de l'homme, 2011.

17. KIRSHEMBLATT-GUIMBLETT Barbara, «Intangible cultural heritage as a meta-cultural production », Museum International, vol. 56, $n^{\circ}$ 1-2, 2004, p. 52-65. 
18. « Folia não é farra ».

19. «Participar da música, assim como participar do ritual como um todo é, em primeiro lugar, um ato de fé, e como tal, é uma fonte de prazer, de satisfação e de alegria que se configura, porém, mais como a realização de um trabalho ou como o cumprimento de uma missão - podendo também envolver sacrifício na forma de sofrimentos pessoais - do que como um divertimento » (LUCAS Glaura, Música e tempo nos rituais do congado mineiro dos Arturos e do Jatobá, Rio de Janeiro, Universidade Federal do Estado do Rio de Janeiro, Thèse de Doctorat, 2005, p. 105.)

20. « A música, organizando a interação coordenada de gestos coletivos ao longo do tempo, concorre de forma significativa para o sentido de união do grupo, para o reforço dos elos de pertencimento à tradição, $e$ para a reafirmação e negociação de valores espirituais, culturais e comunitários. » Ibid., p. 106.

21. « (...) forging a sense of shared experience » REILY Suzel Ana, Voices of the Magi: Enchanted Journeys in Southeast Brazil, Chicago, Chicago University Press, 2002, p. 14.

22. «(...) a folia de reis fisicamente é esforçada, a voz tem que ser muito forte. "

23. « Aí não tem aquele entusiasmo, é fora de época. A gente não recebe nada não, a gente não pede nada, as vezes só da prefeitura o carreto. Entusiasmo não tem por que lá não tem uma pessoa pra receber a folia. A gente foi lá por um motivo de festa, mas o motivo nosso não é esse, não é especificamente uma festa, o motivo nosso é uma alegria na casa de cada um. Quando Jesus andava pelo mundo, ele não pedia para fazer uma festa, ele andava de casa em casa. Eu não gosto de demorar muito tempo numa casa com a folia não, não é o certo, para o nosso conceito não é o certo. »

24. « Nosso patrocínio é nosso peito, nossa vontade, nossa coragem. »

25. «[...] heritages always exist in specific, material forms and are built and noticed by means of sensitive codes - sight, touch, smell, hearing, taste - and not only as abstract formulations that belong to programmatic discourses. [...] exist as material objects situated in certain specific modalities of space, inseparable from the human experience and consequently from the 'body techniques'. In this sense, it is impossible to study 'immaterial heritage' without focusing on its eminently material dimensions, its social and individual uses, as well as its production and effects. » GONÇALVES José Reginaldo, "The Dark Side of the Moon: Heritage, Memory and Place in Rio de Janeiro, Brazil », Vibrant, vol. 10, no 1, 2013, p. 191-192, scielo.br/pdf/vb/v10n1/v10n1a10.pdf (consulté le 1er mai 2019, traduction de l'auteure).

26. CARNEIRO DA CUNHA Manuela, Cultura com aspas e outros ensaios, São Paulo, Cosac Naify, 2009, p. 312. Carneiro da Cunha définit la « culture » comme une catégorie de « ida y vuelta » (aller-retour, va et vient), c'est à dire une catégorie qui «va» en imposant une lecture coloniale des mœurs et pensées des sociétés colonisées et "revient " comme un outil utilisé par les mêmes peuples colonisés pour leur autonomisation et leur résistance à la domination, qui commencent alors à l'utiliser pour défendre leurs modes de vie et leurs droits.

27. BECKER Howard, Les mondes de l'art, Paris, Flammarion, 1998 [1982].

28. HENNION Antoine, La Passion Musicale : Une sociologie de la médiation, Paris, Edition Métailié, 2007 [1993].

29. CAMPos Lúcia, «Sauvegarder une pratique musicale? Une ethnographie du samba de roda à la World Music Expo », Cahiers d'Ethnomusicologie, vol. 24, 2010, p. 141-153.

30. campos Lúcia, Les modes d'écoute d'une poésie chantée : le maracatu de baque solto de la cultura popular à la scène musicale globalisée, Paris, École des Hautes Études en Sciences Sociales, Thèse de Doctorat, 2016.

31. Comme décrit par l'ethnomusicologue Carlos Sandroni dans le texte : "L'ethnomusicologue en médiateur du processus patrimonial : le cas du samba de roda », BORTOLOTTO Chiara (dir.), Le patrimoine culturel immatériel: enjeux d'une nouvelle catégorie, Paris, Éditions de la Maison des sciences de l'homme, 2011.

32. «[...] essa separação foi necessária pra marcar posição: o olhar sobre o patrimônio como processo e não como algo estático " $e$ " a junção da materialidade e imaterialidade vai começar a acontecer a partir das 
ações de salvaguarda e não necessariamente de reconhecimento, pois eu acho que não tem como preservar se você não trabalhar a materialidade e a imaterialidade desse fazer cultural. » Arroyo Michele. Entretien, Belo Horizonte, Brésil, 15 juin 2018.

33. «[...] processos deixaram de contemplar quase que exclusivamente reconstruções do passado para focalizarem manifestações culturais vivas e pulsantes como festas, rituais, saberes, conhecimentos tradicionais"; "esta mudança de estatuto dos bens culturais, ao passarem pelo processo de patrimonialização, vem implicando também em mudanças de percepção, novos trânsitos e a transformação de visões de mundo numa perspectiva dialógica entre diferentes agentes." ABREU Regina, "Patrimonialização das diferenças e os novos sujeitos de direito coletivo no Brasil", TARDY Cécile, DODEBEL Vera (dir.), Memória e Novos Patrimônios, Marseille, OpenEdition Press, 2015, p. 72.

34. "De um lado, vivenciando-o e mantendo a tradição em circulação no grupo e na memória social e, de outro lado, sistematizando-o discursivamente, isto é, representando, por meio de linguagens audiovisuais, fotográficas, digitais, aspectos desta tradição que devem ser permanentes. » Ibid., p. 85.

35. " [...] neste caso, "o bem cultural" não sai de circulação, ou seja, não perde seu "valor de uso" [...]. Mas, efetivamente, ele adquire um valor simbólico que antes não encarnava, mesmo continuando a ser amplamente usado no cotidiano." (ABREU Regina, "Patrimonialização das diferenças e os novos sujeitos de direito coletivo no Brasil", TARDY Cécile, DODEBEL Vera (dir.), Memória e Novos Patrimônios, Marseille, OpenEdition Press, 2015, p. 83.)

36. La présence de professionnels du champ musical (ethnomusicologues, musicologues, pédagogues musicaux) n'a été constatée ni dans les processus de patrimonialisation ni dans les récents recrutements des institutions du patrimoine culturel au Minas Gerais. Concernant le rôle des ethnomusicologues dans les politiques publiques, voir: LÜHNING Angela, TUGNY Rosângela (dir.), Etnomusicologia no Brasil, Salvador, EDUFBA, 2016.

\section{RÉSUMÉS}

Cet article porte sur la patrimonialisation de la folia de reis, pratique religieuse et musicale du Minas Gerais (Brésil). Il met l'accent sur les articulations et décalages entre les groupes de folias de reis et les politiques du patrimoine culturel immatériel quant à leurs conceptions et usages respectifs de la musique. Une ethnographie de la Folia de Reis do Campo Belo met au jour les liens entre musique, religion et mémoire à travers lesquels les foliões conçoivent leur patrimoine. C'est en revanche le paradigme de la «musique-objet» qui prévaut dans le cadre des politiques publiques, en phase avec les paramètres de la production culturelle conventionnelle : les formats de présentation sont survalorisés, et l'un des instruments en jeu, la viola, déclaré «patrimoine culturel immatériel », entretient avec la pratique de la folia une relation métonymique. Dès lors, il convient d'analyser l'articulation entre matériel et immatériel dans le processus de patrimonialisation. Notre conclusion souligne quant à elle la dimension participative des politiques de sauvegarde du patrimoine immatériel, ainsi que le défi que représente la valorisation du patrimoine au quotidien à travers l'éducation et les recherches collaboratives.

This article discusses the heritagisation of folia de reis, a religious and musical practice in Minas Gerais (Brazil). It focuses on the articulations and gaps between the folias de reis groups and the ICH policies in terms of their respective conceptions and uses of the music. An ethnography on the Folia de Reis do Campo Belo reveals the connections between music, religion and memory through which the folioes conceive their heritage. Yet it is the "music-object" paradigm that 
prevails in the public policies, in line with the parameters of conventional cultural production: the performance formats are glorified, and one of the instruments, the viola, declared "intangible cultural heritage", has a metonymic relationship with the practice of folia. The aim, therefore, is to examine the intersection of the tangible and intangible in the heritagisation process. Our conclusion points out the participatory dimension of the intangible heritage protection policies, and the challenge of promoting the appreciation of heritage in everyday life through education and collaborative research.

\section{INDEX}

Mots-clés : patrimoine culturel immatériel, folia de reis, ethnographie, Minas Gerais

Keywords : intangible cultural heritage, folia de reis, ethnography, Minas Gerais

\section{AUTEUR}

\section{LÚCIA CAMPOS}

Lúcia Campos est professeure et chercheuse à l'Université de l'État du Minas Gerais (UEMG). Son master en anthropologie et son doctorat « Musique, Histoire, Société » de l'EHESS (2016) ont porté sur les relations que les groupes brésiliens de « cultura popular » entretiennent avec les politiques publiques et avec la scène musicale, à partir d'études ethnographiques sur le maracatu de baque solto, la ciranda et le samba de roda. À la faveur d'un post-doctorat (PNPD - Capes) à l'Université Fédérale du Minas Gerais (UFMG) portant notamment sur la folia de reis, elle a étudié les politiques de sauvegarde du Patrimoine Culturel Immatériel au Brésil. Elle a publié dans les Cahiers d'ethnomusicologie, dans le Yearbook for Traditional Music ainsi que dans des ouvrages collectifs. Elle a coordonné Territórios de Invenção : por uma formação musical expandida (2017) et Territórios de Invenção : à escuta (2019), et prépare actuellement la publication de sa thèse de doctorat. 